\title{
A Unify Method for Solving Quadratic Equations
}

\author{
Okoli, O.C. \\ Department of Mathematics, Chukwuemeka Odumegwu Ojukwu University, Anambra State, \\ Nigeria.
}

odicomatics@yahoo.com,odico2003@yahoo.com; +234(0)8036941434

\begin{abstract}
Let $a x^{2}+b x+c=0$ be a quadratic equation in one variable, before now, Graphical method, Factorization method, Completing the square method and Formula method (the so-called almighty formula) are commonly used for solving quadratic equations that have a closed form solution. In this work, we introduced a unify approach called the Two-Step formula method for solving quadratic equations, which gives a faster method of solution; $x=\frac{-q}{a}$ or $x=\frac{-p}{a}$ for cases where the two numbers $p$ and $q$ satisfying some required conditions can be easily chosen.
\end{abstract}

Original Research Article

Received: 30th Sept., 2017.

Accepted: 15th Oct, 2017.

Published: 23 ${ }^{\text {rd }}$ Oct., 2017.

Keywords:

Quadratic equation; Polyno-

mial; Factorization; Two step

method.

\subsection{INTRODUCTION.}

$\mathrm{P}$ olynomial equations practically appears in different branches of study, physics, chemistry, economics etc. The general form of a polynomial equation of degree $n$ is given by

$$
a_{n} x^{n}+a_{n-1} x^{n-1}+\ldots+a_{3} x^{3}+a_{2} x^{2}+a_{1} x+a_{0}=0 ; \quad a_{n} \neq 0---
$$

Where $a_{j} ; j=0,1,2,3, \ldots, n$ are the constant coefficients of the unknown variable $x$.

If $n=1$ and $a_{1} \neq 0$, the polynomial equation of degree $n$ in 1.1 takes a special name known as LINEAR EQUATION which is given by

$$
a_{1} x+a_{0}=0 ; \quad a_{1} \neq 0---(1.2)
$$

If $n=2$ and $a_{2} \neq 0$, the polynomial equation of degree $n$ in 1.1 takes a special name known as QUADRATIC EQUATION which is given by

$$
a_{2} x^{2}+a_{1} x+a_{0}=0 ; \quad a_{2} \neq 0---(1.3)
$$

If $n=3$ and $a_{3} \neq 0$, the polynomial equation of degree $n$ in 4.1 takes a special name known as CUBIC EQUATION which is given by

$$
a_{3} x^{3}+a_{2} x^{2}+a_{1} x+a_{0}=0 ; \quad a_{3} \neq 0---(1.4)
$$

For the purpose of our study, we shall restrict our study for the case where $n \leq 2$. The results expressed in this work shall be of immense benefit to students interested in quadratic equations who has yearn for alternative approach for solving this equations. In order to incite the reader we begin with the very fundamentals. 


\subsection{METHODS OF SOLUTION TO QUADRATIC EQUATIONS}

There are several approaches/methods to solutions of a quadratic equation as contained in most standard text books (K. A. Stroud Booth D. J. (2007)), such methods includes:

1. Factorization method

2. Completing the square method

3. Formula method

4. Graphical method

Recall that the general form of a quadratic equation (as in equation 1.3) is given by $a_{2} x^{2}+a_{1} x+a_{0}=0$. Without any loss of generality and for simplicity, we shall henceforth write this general quadratic equation as $a x^{2}+b x+$ $c=0$, where $a, b, c$ are elements of real numbers. We shall proceed by illustrate with telling examples on these methods mentioned above. For further study on this subject, interested reader my consult the references therein.

\subsubsection{FACTORIZATION METHOD}

Example 1 Find the value of $x$ in the following equations using factorization method

(i) $2 x^{2}+5 x-12=0$ (ii) $x^{2}-3 x-28=0$ (iii) $x^{2}-9 x+20=0$ (iv) $4 x^{2}-2 x-2=0$

\section{SOLUTION}

(i) $2 x^{2}+5 x-12=0$.

If we choose $p=+8$ and $q=-3$ then one can check to see that $p+q=b$ and $p q=a c$. Thus

$2 x^{2}+5 x-12=2 x^{2}+(8-3) x-12=2 x^{2}+8 x-3 x-12=\left(2 x^{2}+8 x\right)-(3 x+12)=$

$2 x(x+4)-3(x+4)=(2 x-3)(x+4)$

$\therefore(2 x-3)(x+4)=0 ; \Rightarrow 2 x-3=0$ or $x+4=0 ; \Rightarrow x=\frac{3}{2}$ or $x=4$

(ii) $x^{2}-3 x-28=0$.

If we choose $p=-7$ and $q=4$ then one can check to see that $p+q=b$ and $p q=a c$. Thus $x^{2}-3 x-28=x^{2}+(-7+4) x-28=x^{2}-7 x+4 x-28=\left(x^{2}-7 x\right)+(4 x-28)=$ $x(x-7)+4(x-7)=(x+4)(x-7)$

$\therefore(x+4)(x-7)=0 ; \Rightarrow x+4=0$ or $x-7=0 ; \Rightarrow x=-4$ or $x=7$.

(iii) $x^{2}-9 x+20=0$.

If we choose $p=-5$ and $q=-4$ then one can check to see that $p+q=b$ and $p q=a c$. Thus $x^{2}-9 x+20=x^{2}+(-5-4) x+20=x^{2}-5 x-4 x+20=\left(x^{2}-5 x\right)-(4 x-20)=$ $x(x-5)-4(x-5)=(x-4)(x-5)$

$\therefore(x-4)(x-5)=0 ; \rightarrow x-4=0$ or $x-5=0 ; \rightarrow x=4$ or $x=5$

(iv) $4 x^{2}-2 x-2=0$

If we choose $p=-4$ and $q=2$ then one can check to see that $p+q=b$ and $p q=a c$. Thus $4 x^{2}-2 x-2=4 x^{2}+(-4+2) x-2=4 x^{2}-4 x+2 x-2=\left(4 x^{2}-4 x\right)+(2 x-2)=$ $4 x(x-1)+2(x-1)=(4 x+2)(x-1)$.

$$
\therefore(4 x+2)(x-1)=0 ; \Rightarrow 4 x+2=0 \text { or } x-1=0 ; \Rightarrow x=\frac{-1}{2} \text { or } x=1
$$




\subsubsection{COMPLETING THE SQUARE METHOD}

Completing the square method, technically in this method one is requires to make $x$ the subject of formula in the quadratic equation. This is some worth equivalent to the quadratic formula m method. The only different is that in completing the square method we solve to make $x$ the subject formula and then simplify to get the value of $x$ while in quadratic formula method $x$ is already a subject formula, all we need do is to substitute appropriately into the formula and simplify. The procedure for completing square method is as follows;

Consider the quadratic equation $a x^{2}+b x+c=0$

divid both side by $a: \quad x^{2}+\frac{b}{a} x+\frac{c}{a}=0$;

$$
\Rightarrow x^{2}+\frac{b}{a} x=-\frac{c}{a}
$$

We now divide the coefficient of $x\left(\right.$ i.e. $\left.\frac{b}{a}\right)$ by $2\left(\right.$ i.e. $\left.\frac{b}{2 a}\right)$ and square the resulting number $\left(i . e .\left(\frac{b}{2 a}\right)^{2}\right)$. We add this resulting number to both side of the equations to obtain

$$
\begin{aligned}
& x^{2}+\frac{b}{a} x+\left(\frac{b}{2 a}\right)^{2}=-\frac{c}{a}+\left(\frac{b}{2 a}\right)^{2} ; \Rightarrow\left(x+\frac{b}{2 a}\right)^{2}=-\frac{c}{a}+\left(\frac{b}{2 a}\right)^{2} ; \\
& \Rightarrow x+\frac{b}{2 a}= \pm \sqrt{\left(\frac{b}{2 a}\right)^{2}-\frac{c}{a}} ; \Rightarrow x=-\frac{b}{2 a} \pm \sqrt{\left(\frac{b}{2 a}\right)^{2}-\frac{c}{a}} ; \Rightarrow x=\frac{-b \pm \sqrt{b^{2}-4 a c}}{2 a}
\end{aligned}
$$

If we substitute for the values of $a, b, c$ and simplify the right hand side (RHS) we obtain the values of $x$ as required. This is the completing square method. By this, one can easily see that the completing square method implies the quadratic formula method which we shall treat in the subsequent section.

Example 2 Find the value(s) of $x$ in the following equations using completing the square method.

(i) $2 x^{2}+5 x-12=0$ (ii) $x^{2}-3 x-28=0$

\section{SOLUTION.}

(i) $2 x^{2}+5 x-12=0 ; \Rightarrow x^{2}+\frac{5}{2} x-6=0 ; \Rightarrow x^{2}+\frac{5}{2} x=6 ; \Rightarrow x^{2}+\frac{5}{2} x+\left(\frac{5}{4}\right)^{2}=6+\left(\frac{5}{4}\right)^{2}$;

$\Rightarrow\left(x+\frac{5}{4}\right)^{2}=6+\frac{25}{16} ; \Rightarrow\left(x+\frac{5}{4}\right)^{2}=\frac{96+25}{16} ; \Rightarrow\left(x+\frac{5}{4}\right)^{2}=\frac{121}{16} ; \Rightarrow x+\frac{5}{4}= \pm \sqrt{\frac{121}{16}}$;

$\Rightarrow x+\frac{5}{4}= \pm \frac{11}{4} ; \Rightarrow x=-\frac{5}{4} \pm \frac{11}{4} ; \Rightarrow x=\left(-\frac{5}{4}-\frac{11}{4}\right)$ or $\left(-\frac{5}{4}+\frac{11}{4}\right)$;

$\Rightarrow x=\left(-\frac{16}{4}\right)$ or $\left(\frac{6}{4}\right) ; x=-4$ or $\frac{3}{2}$

(ii) $x^{2}-3 x-28=0 ; \Rightarrow x^{2}-3 x=28 ; \Rightarrow x^{2}-3 x+\left(\frac{3}{2}\right)^{2}=28+\left(\frac{3}{2}\right)^{2}$;

$\Rightarrow\left(x-\frac{3}{2}\right)^{2}=28+\frac{9}{4} ; \Rightarrow\left(x-\frac{3}{2}\right)^{2}=\frac{112+9}{4} ; \Rightarrow\left(x-\frac{3}{2}\right)^{2}=\frac{121}{4} ; \Rightarrow x-\frac{3}{2}= \pm \sqrt{\frac{121}{4}}$;

$\Rightarrow x-\frac{3}{2}= \pm \frac{11}{2} ; \Rightarrow x=\frac{3}{2} \pm \frac{11}{2} ; \Rightarrow x=\left(\frac{3}{2}-\frac{11}{2}\right)$ or $\left(\frac{3}{2}+\frac{11}{2}\right)$;

$\Rightarrow x=\left(-\frac{8}{2}\right)$ or $\left(\frac{14}{2}\right) ; x=-4$ or 7

\subsubsection{QUADRATIC FORMULA METHOD}

In quadratic formula method we simply invoke the formula obtained from the completing square method above which is given by

$$
x=\frac{-b \pm \sqrt{b^{2}-4 a c}}{2 a}
$$


Example 3 Find the value(s) of $x$ in the following equations using the formula method.

(i) $2 x^{2}+5 x-12=0$ (ii) $x^{2}-3 x-28=0$

\section{SOLUTION.}

(i) $x^{2}-9 x+20=0$; then $a=1, b=-9, c=20$ Substituting this into the quadratic formula we have

$x=\frac{-b \pm \sqrt{b^{2}-4 a c}}{2 a}=\frac{-(-9) \pm \sqrt{(-9)^{2}-4 \times 1 \times 20}}{2 \times 1}=\frac{9 \pm \sqrt{81-80}}{2}=\frac{9 \pm \sqrt{1}}{2}=\frac{9 \pm 1}{2} ; \Rightarrow x=\frac{10}{2}$ or $x=\frac{8}{2}$;

$\Rightarrow x=5$ or $x=4$

(ii) $4 x^{2}-2 x-2=0$; then $a=4, b=-2, c=-2$ Substituting this into the quadratic formula we have

$x=\frac{-b \pm \sqrt{b^{2}-4 a c}}{2 a}=\frac{-(-2) \pm \sqrt{(-2)^{2}-4 \times 4 \times(-2)}}{2 \times 4}=\frac{2 \pm \sqrt{4+32}}{8}=\frac{2 \pm \sqrt{36}}{8}=\frac{2 \pm 6}{8}$

$\Rightarrow x=\frac{8}{8}$ or $x=\frac{-4}{8} ; \Rightarrow x=1$ or $x=\frac{-1}{2}$

\section{MAIN RESULTS}

In this section we introduce the main result(s) of this work, which is the method of Two-Step Formula for solving quadratic equation. We shall now begin as follows.

\subsection{TWO-STEP FORMULA METHOD}

The Two-Step Formula Method is a unifying method for both the factorization method and the completing the square method, haven seen that completing the square method implies the quadratic formula method. We shall state and prove the theorem(s) governing this claim in the sequel. Now observe that in factorization method, one is technically required to guess for two numbers $p$ and $q$ satisfying certain conditions; this condition give rise to what is often referred to as One-Linear One-Quadratic Simultaneous Equations. To introduce this method, we proceed by proving the following theorems.

\section{Theorem 3.1}

Given the quadratic equation $a x^{2}+b x+c=0$, suppose there exists numbers $p$ and $q$ satisfying the equations

$$
\begin{gathered}
p+q=b----(1.5) \\
p q=a c----(1.6)
\end{gathered}
$$

then $x=\frac{-q}{a}$ or $x=\frac{-p}{a}$ is a solution to the quadratic equation.

Proof.

By hypothesis equation (1.5) and equation (1.6) are satisfy, then

$$
\begin{gathered}
a x^{2}+b x+c=0 ; \Rightarrow a x^{2}+(p+q) x+\frac{p q}{a}=0 ; \Rightarrow(a x)^{2}+a(p+q) x+p q=0 \\
\Rightarrow(a x)^{2}+a x p+a x q+p q=0 ; \Rightarrow\left((a x)^{2}+a x p\right)+(a x q+p q)=0 \\
\Rightarrow a x(a x+p)+q(a x+p)=0 ; \Rightarrow(a x+q)(a x+p)=0 \\
\Rightarrow(a x+q)=0 \text { or }(a x+p)=0 \\
\Rightarrow a x=-q \text { or } a x=-p ; \Rightarrow x=\frac{-q}{a} \text { or } x=\frac{-p}{a}
\end{gathered}
$$

This complete the proof as required.

This is a solution to the quadratic equation $a x^{2}+b x+c=0$. Hence we summaries the two-step formula method in the following three line equations

Given $a x^{2}+b x+c=0$, if there exist $p$ and $q$ such that

$$
p+q=b
$$




$$
\begin{gathered}
p q=a c \\
\text { then } x=\frac{-q}{a} \text { or } x=\frac{-p}{a}
\end{gathered}
$$

We will illustrate this method with the same problems we solved above.

Example 4 Find the value of $x$ in the following equations using two-step formula method

(i) $2 x^{2}+5 x-12=0$ (ii) $x^{2}-3 x-28=0$ (iii) $x^{2}-9 x+20=0$ (iv) $4 x^{2}-2 x-2=0$

\section{SOLUTION.}

(i) $2 x^{2}+5 x-12=0$.

Observe that $a=2, b=5, c=-12$, if we choose $p=+8$ and $q=-3$ then one can check to see that $p+q=$ $b$ and $p q=a c$. Then

$x=\frac{-q}{a}=\frac{-(-3)}{2}=\frac{3}{2}$ or $x=\frac{-p}{a}=\frac{-(8)}{2}=-4$

(ii) $x^{2}-3 x-28=0$.

Observe that $a=1, b=-3, c=-28$, if we choose $p=-7$ and $q=4$ then one can check to see that $p+q=$ $b$ and $p q=a c$. Then

$x=\frac{-q}{a}=\frac{-(4)}{1}=-4$ or $x=\frac{-p}{a}=\frac{-(-7)}{1}=7$

(iii) $x^{2}-9 x+20=0$.

Observe that $a=1, b=-9, c=+20$, if we choose $p=-5$ and $q=-4$ then one can check to see that $p+$ $q=b$ and $p q=a c$. Then

$x=\frac{-q}{a}=\frac{-(-4)}{1}=4$ or $x=\frac{-p}{a}=\frac{-(-5)}{1}=5$

(iv) $4 x^{2}-2 x-2=0$

Observe that $a=4, b=-2, c=-2$, if we choose $p=-4$ and $q=2$ then one can check to see that $p+q=b$ and $p q=a c$. Then

$x=\frac{-q}{a}=\frac{-(2)}{4}=\frac{-1}{2}$ or $x=\frac{-p}{a}=\frac{-(-4)}{4}=1$.

\subsection{CONSEQUENCES OF TWO-STEP FORMULA METHOD}

It is worth mentioning that if it becomes difficult to guess correctly the values of $p$ and $q$, then we can obtain an equivalent quadratic equation by combining equation (1.5) and equation (1.6); whose solution provides answer for the values of $p$ and $q$.

We often time resort to the quadratic formula method when the factorization method become difficult to apply directly. That is if it became difficult to guess $p$ and $q$ for any given quadratic equation $a x^{2}+b x+c=0$. For such situations we solve the One-Linear One-Quadratic Simultaneous Equations for $p$ and $q$ in equation (1.5) and equation (1.6). We now proceed to prove that the conditions prescribed by theorem 3.1 implies the quadratic formula method.

\section{Theorem 3.2}

Given the quadratic equation $a x^{2}+b x+c=0$, suppose equation (1.5) and equation (1.6) are satisfied, then

$$
x=\frac{-q}{a} \text { or } x=\frac{-p}{a}---(1.7)
$$

implies the quadratic formula method

\section{Proof.}

In equation (1.5) if we make $q$ the subject (i.e. $q=b-p$ ) and substitute into equation (1.6) we obtain $p(b-p)=a c$. 


$$
\Rightarrow p^{2}-b p+a c=0-
$$

Which is a quadratic equation in $p$. Now we solve for $p$ (using the completing square method) as follows;

$$
\begin{aligned}
& \Rightarrow p^{2}-b p+a c=0 ; \Rightarrow p^{2}-b p=-a c ; \Rightarrow p^{2}-b p+\left(-\frac{b}{2}\right)^{2}=-a c+\left(-\frac{b}{2}\right)^{2} ; \\
& \Rightarrow\left(p-\frac{b}{2}\right)^{2}=-a c+\frac{b^{2}}{4} ; \Rightarrow\left(p-\frac{b}{2}\right)^{2}=\frac{b^{2}-4 a c}{4} ; \Rightarrow p-\frac{b}{2}= \pm \sqrt{\frac{b^{2}-4 a c}{4}} ; \\
& \Rightarrow p=\frac{b}{2} \pm \frac{\sqrt{b^{2}-4 a c}}{2} ; \Rightarrow p=\frac{b \pm \sqrt{b^{2}-4 a c}}{2} \text {. So that } q=b-p=b-\left(\frac{b \pm \sqrt{b^{2}-4 a c}}{2}\right)=\frac{b \pm \sqrt{b^{2}-4 a c}}{2}
\end{aligned}
$$

To obtain the value of $x$ we substitute for $p$ and $q$ in equation (1.7) as follows;

$$
\begin{gathered}
x=\frac{-q}{a}=\frac{-\left(\frac{b \pm \sqrt{b^{2}-4 a c}}{2}\right)}{a}=\frac{-b \pm \sqrt{b^{2}-4 a c}}{2 a} \quad \text { or } \quad x=\frac{-p}{a}=\frac{-\left(\frac{b \pm \sqrt{b^{2}-4 a c}}{2}\right)}{a}=\frac{-b \pm \sqrt{b^{2}-4 a c}}{2 a} \\
\Rightarrow x=\frac{-b \pm \sqrt{b^{2}-4 a c}}{2 a}
\end{gathered}
$$

Which is the quadratic formula. This completes the proof.

\subsection{CONCLUSION}

So far the Two-Step Formula Method introduced for solving quadratic equation unifies other existing methods. Except for graphical method, we have basically the factorization method and quadratic formula method for solving quadratic equation from time immemorial. We have already seen that completing the square method is equivalent to the quadratic formula method as illustrated above. Furthermore, we stated and proved some theorem(s) which reveals that if:

1. The two numbers $p$ and $q$ satisfying the required conditions can be chosen appropriately, then the solution to the quadratic equation is simply $x=\frac{-q}{a}$ or $x=\frac{-p}{a}$, which is straight to the point (see example 4 above) when compared to the usual factorization process/method employed in example 1 above.

2. It became difficult to guess $p$ and $q$ for any given quadratic equation $a x^{2}+b x+c=0$. For such situations we solve the One-Linear One-Quadratic Simultaneous Equations for $p$ and $q$ in equation (1.5) and equation (1.6) whose solution provides answer for the values of $p$ and $q$, so that the solution to the quadratic equation is simply $x=\frac{-q}{a}$ or $x=\frac{-p}{a}$. And that the conditions prescribed by theorem 3.1 implies the quadratic formula method.

\section{REFERENCES}

Adewumi, M. (2014). Solution techniques for cubic expressions and root finding. Penn State University Courseware Module.

Bessels, S. (2006). One step beyond the solvable equation. www.staff.science.uu.nc/.../Afstudeerscriptie_sander_Bessels.pdf.

Dickson, L.E. (1914); Elementary theory of equations. New York: John Wiley and sons.

Hahn, K. (2005). Solving cubic and quartic polynomials.
www.Karlcalculus.org/pdf/cubicquartic.pdf.

Mukundan, T.R. (2010); Solution of cubic equations: An alternative method. Resonance, 15:

347-350. https://doi.org/10.1007/s12045-010-0028-2.

Stroud, K.A. and Booth, D.J. (2007); Engineering mathematics. London: Palgrave Macmilan, 2007.

Weisstein, E.W. Cubic Formular. www.mathworld.wolf$\underline{\text { ram.com/CubicFormular.html. }}$

\section{$\underline{\text { How to cite this article }}$}

Okoli O.C. (2017). A Unify Method for Solving Quadratic Equations. Tropical Journal of Applied Natural Sciences, 2(1): 7883. Doi: https://doi.org/10.25240/TJANS.2017.2.1.13. 